\title{
Particle separation in surface acoustic wave microfluidic devices using reprogrammable, pseudo-standing waves
}

Cite as: Appl. Phys. Lett. 113, 044101 (2018); https://doi.org/10.1063/1.5035261

Submitted: 13 April 2018 . Accepted: 09 July 2018 . Published Online: 23 July 2018

(D) Gergely Simon, Yan Pailhas, (D) Marco A. B. Andrade, (D) Julien Reboud, Jose Marques-Hueso, (D) Marc P. Y. Desmulliez, (D) Jonathan M. Cooper, (D) Mathis O. Riehle, and (D) Anne L. Bernassau

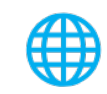

\section{ARTICLES YOU MAY BE INTERESTED IN}

Particle separation by phase modulated surface acoustic waves

Biomicrofluidics 11, 054115 (2017); https://doi.org/10.1063/1.5001998

Patterning and manipulating microparticles into a three-dimensional matrix using standing surface acoustic waves

Applied Physics Letters 112, 213507 (2018); https://doi.org/10.1063/1.5024888

Binary particle separation in droplet microfluidics using acoustophoresis

Applied Physics Letters 112, 063701 (2018); https://doi.org/10.1063/1.5020356

\section{Hall Effect Measurement Handbook}

A comprehensive resource for researchers Explore theory, methods, sources of errors, and ways to minimize the effects of errors

\section{Request it here}

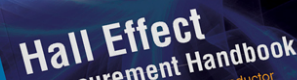

Measurement Hand

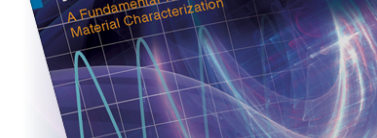

Lakeshore
cRYOTRONICS 


\title{
Particle separation in surface acoustic wave microfluidic devices using reprogrammable, pseudo-standing waves
}

\author{
Gergely Simon, ${ }^{1}$ Yan Pailhas, ${ }^{2}$ Marco A. B. Andrade, ${ }^{3}$ Julien Reboud, ${ }^{4}$ \\ Jose Marques-Hueso, ${ }^{1}$ Marc P. Y. Desmulliez, ${ }^{1}$ Jonathan M. Cooper, ${ }^{4}$ Mathis O. Riehle, ${ }^{5}$ \\ and Anne L. Bernassau ${ }^{1}$ \\ ${ }^{1}$ School of Engineering and Physical Sciences, Heriot-Watt University, Edinburgh, United Kingdom \\ ${ }^{2}$ Centre for Maritime Research and Experimentation, La Spezia, Italy \\ ${ }^{3}$ Institute of Physics, University of São Paulo, São Paulo, Brazil \\ ${ }^{4}$ School of Engineering, University of Glasgow, Glasgow, United Kingdom \\ ${ }^{5}$ Institute of Molecular Cell and Systems Biology, Centre for Cell Engineering, University of Glasgow, \\ Glasgow, United Kingdom
}

(Received 13 April 2018; accepted 9 July 2018; published online 23 July 2018)

\begin{abstract}
We report size and density/compressibility-based particle sorting using on-off quasi-standing waves based on the frequency difference between two ultrasonic transducers. The $13.3 \mathrm{MHz}$ fundamental operating frequency of the surface acoustic wave microfluidic device allows the manipulation of particles on the micrometer scale. Experiments, validated by computational fluid dynamics, were carried out to demonstrate size-based sorting of 5-14.5 $\mu \mathrm{m}$ diameter polystyrene (PS) particles and density/compressibility-based sorting of $10 \mu \mathrm{m}$ PS, iron-oxide, and poly(methyl methacrylate) particles, with densities ranging from 1.05 to $1.5 \mathrm{~g} / \mathrm{cm}^{3}$. The method shows a sorting efficiency of $>90 \%$ and a purity of $>80 \%$ for particle separation of $10 \mu \mathrm{m}$ and $14.5 \mu \mathrm{m}$, demonstrating better performance than similar sorting methods recently published (72\%-83\% efficiency). The sorting technique demonstrates high selectivity separation of particles, with the smallest particle ratio being 1.33 , compared to 2.5 in previous work. Density/compressibility-based sorting of polystyrene and iron-oxide particles showed an efficiency of $97 \pm 4 \%$ and a purity of $91 \pm 5 \%$. By varying the sign of the acoustic excitation signal, continuous batch acoustic sorting of target particles to a desired outlet was demonstrated with good sorting stability against variations of the inflow rates. Published by AIP Publishing. https://doi.org/10.1063/1.5035261
\end{abstract}

Particle or living cell separation is a critical enabling step in industrial, chemical, and biomedical processes. ${ }^{1}$ Acoustic separation techniques are especially advantageous for their non-contact, label-free, biocompatible properties. ${ }^{2}$ To achieve particle sorting, most acoustic separation methods utilize the difference in time-of-flight of particles subjected to a standing pressure wave and therefore are limited to separation distances up to a quarter of the wavelength. ${ }^{3}$ Transducers tilted with respect to the axis of the separation channel alleviate the limited separation distance, but the translational distance of particles is still bound by the geometrical design. ${ }^{4}$

Particles can also be translated unconstrained by a quasi-standing wave, as a result of phase modulation or by a small frequency difference between opposing transducers. ${ }^{5-7}$ In both techniques, the maximum translational speed of the particles has a size-dependence that can be utilized for sorting. ${ }^{8}$ Recently, a continuously frequency modulated acoustic field method was applied in a surface acoustic wave (SAW) device. ${ }^{9}$ However, in this technique, the particle movement is fluctuating, demonstrating limited selectivity (2.5-fold or greater diameter ratio) and efficiency (up to $83 \%$ ). We propose a modified method that increases both selectivity and efficiency to open up a wide range of applications in biomedical engineering and beyond.

Our previous works used phase modulation to achieve particle sorting. ${ }^{10,11}$ These were carried out in the absence of the flow, and sorting had been presented only in a single direction. Moreover, phase modulation requires a more complex control signal than the frequency modulation technique presented in this letter.

In this letter, we extend the existing knowledge on particle separation using quasi-standing waves and apply the method for particle separation in surface wave devices. Average particle speed measurements were carried out for various frequency differences, $\Delta f$, ranging from -1.6 to $1.6 \mathrm{~Hz}$. Within this interval, the particles either linearly translate with the moving pressure node (below maximum particle velocity) or are dominated by an oscillatory motion (above maximum speed). We provide a theoretical framework to allow adaptation of the design parameters to different geometries. This technique provides the possibility of steering target particles to different outlets by adjusting the flow rates and the sign of the modulation signal. We term these "upwards" (target exit at the top outlet) and "downwards" (target exit at the bottom outlet) sorting.

The schematic of the sorting device is shown in Fig. 1. The transducers have a central frequency of $13.3 \mathrm{MHz}$, corresponding to $\lambda=300 \mu \mathrm{m}$ for a 128 -Y cut X-oriented lithium niobate substrate. ${ }^{3}$ The polydimethylsiloxane (PDMS) channel has a width of $240 \mu \mathrm{m}$, allowing for two acoustic pressure nodes. The desired symmetric pressure node distribution within the device can be achieved by changing the relative phase of the acoustic waves propagated by the transducers. ${ }^{12}$ More details on device fabrication and experimental setup have been presented elsewhere. ${ }^{11}$ The microfluidic 


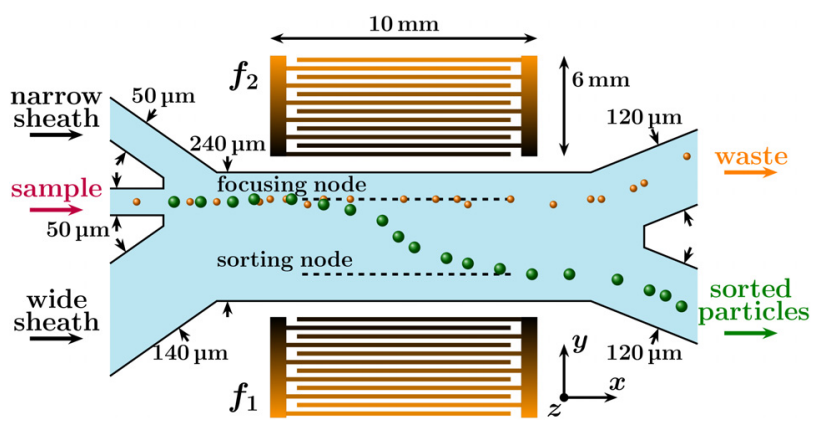

FIG. 1. Schematic of the device and illustration of sorting.

device has an asymmetric inlet configuration: one sheath and sample inlet are $50 \mu \mathrm{m}$ wide, while another sheath inlet is $140 \mu \mathrm{m}$ wide. The flow rates at the three inlet channels are adjusted to focus the particles at one of the pressure nodes, termed the "focusing" node (Fig. 1). When the frequency difference, $\Delta f$, is created between the two transducers, the interplay between acoustic radiation and drag forces selectively displaces the larger particles towards the other pressure node, defined here as "sorting" node. An asymmetric inlet configuration is designed to investigate how significant particle focusing is on the efficiency and purity on "upwards" and "downwards" sorting.

Two opposing transducers activated at the same frequency result in a standing wave field, where the acoustic radiation force collects particles at either the nodes or antinodes. ${ }^{13}$ When the two frequencies, $f_{1}$ and $f_{2}$, differ slightly, the resulting standing wave moves spatially with speed

$$
v_{\mathrm{p}}=2 \pi\left(f_{1}-f_{2}\right) /\left(k_{y, 1}+k_{y, 2}\right)=2 \pi \Delta f /\left(k_{y, 1}+k_{y, 2}\right),
$$

where $k_{y, i}=2 \pi / \lambda_{i}$ with $\lambda_{i}=c / f_{i}$, and $c$ being the surface wave velocity, meaning that the pressure nodes and therefore the particles are always displaced away from the higher frequency transducer. ${ }^{5}$ Assuming that $\Delta f \ll f_{0}$ and therefore $k_{y, 1} \approx k_{y, 2}=2 \pi f_{0} / c$, where $f_{0}=13.3 \mathrm{MHz}$ is the central frequency of the transducer and $c \approx 3990 \mathrm{~ms}^{-1}$, the translational speed is

$$
v_{\mathrm{p}}=150 \cdot \Delta f \text { in } \mu \mathrm{m} / \mathrm{s} .
$$

The acoustic radiation force can be obtained from the pressure distribution (see supplementary material)

$$
F_{\mathrm{ac}, y}=-c_{\mathrm{ac}} \sin \left(2 k_{y} y-2 \pi \Delta f t\right),
$$

where all particle and medium dependent parameters are included in $c_{\mathrm{ac}}$, and $k_{y} \approx 2 \pi f_{0} / c$. As the particle is placed in a liquid medium, the Stokes' drag force opposes the acoustic radiation force as a result of the inertial approximation ${ }^{14}$

$$
-F_{\mathrm{ac}, y}=F_{\mathrm{drag}}=-6 \pi \eta R \dot{y}=-c_{\mathrm{visc}} \dot{y},
$$

where $R$ is the particle radius, $\dot{y}$ is the relative speed of the particle with respect to the medium, and $\eta$ is the dynamic viscosity of the medium. Wall effects of the channel can be incorporated into the viscosity. ${ }^{15}$ This equation can be used to obtain particle trajectories (see supplementary material).

Both the trajectory equation and the force balance predict a limit for the linear translation of particles. The maximum particle speed is obtained from the maximum radiation force ${ }^{5}$

$$
v_{\max }=(\dot{y})_{\max }=c_{\mathrm{ac}} / c_{\mathrm{visc}} .
$$

Any frequency difference that causes a nodal translational speed $v_{\mathrm{p}}$ less than $v_{\max }$ forces the particles to move linearly with a constant speed. However, if the nodal speed is greater than the maximum speed $\left(v_{\mathrm{p}}>v_{\max }\right)$, the particles oscillate and shift at the same time, in a less deterministic manner.

Speed measurements were carried out to demonstrate this phenomenon. Particle trajectories were recorded to calculate average particle speeds. The results for $19 \mathrm{~V}_{\mathrm{pk}-\mathrm{pk}}$ transducer voltage for 10 and $14.5 \mu \mathrm{m}$ particles are shown in Fig. 2. For frequency differences between -0.4 and $0.4 \mathrm{~Hz}$ (Fig. 2, Region I), both particles are below their respective speed limit $v_{\mathrm{p}}<v_{\max }$, so they both translate simultaneously, ${ }^{5}$ and no sorting can be achieved. When the frequency difference is less than -0.85 or greater than $0.85 \mathrm{~Hz}$ (Fig. 2, Regions III), as $v_{\mathrm{p}}>v_{\max }$, both particles only oscillate with small average speeds, which cannot be used for sorting. However, in regions between -0.85 to -0.4 and 0.4 to $0.85 \mathrm{~Hz}$ (Fig. 2, Regions II), the large particles are below their maximum speed and can be translated linearly, while the small particles oscillate and shift with a lower average speed. These regions are promising for sorting applications. The regions from -0.8 to $-0.4 \mathrm{~Hz}$ and from 0.4 to $0.8 \mathrm{~Hz}$ are defined as the downwards and upwards regimes when target particles exit via the lower and upper outlets, respectively. Although the frequency difference between transducers is six orders of magnitude smaller than the center frequency, speed measurements and separation experiments were highly reproducible showing good performance of the technique.

To utilize the sorting regions (Fig. 2, Regions II), we apply to both transducers the frequency pattern shown in Figs. 3(a) and 3(b). The frequency difference, $\Delta f$, is switched

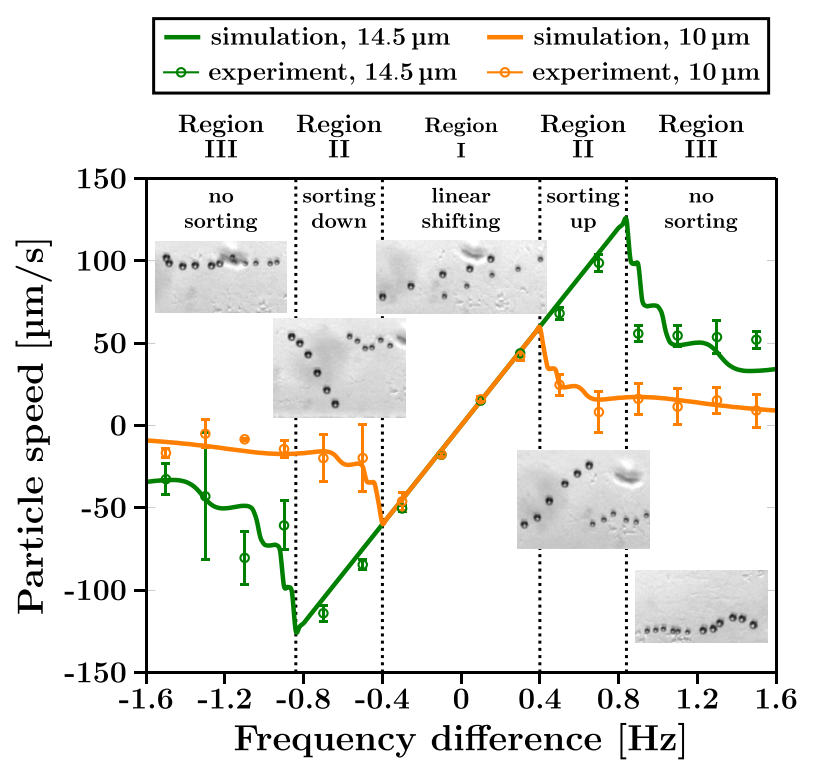

FIG. 2. Experimental and theoretical average particle speed for various frequency difference values between transducers. Insets are overlay images of the corresponding videos, illustrating particle motion. 
on for a period of time $t_{\mathrm{ON}}$, followed by an off period, $t_{\mathrm{OFF}}$. Note that the transducers are on for the entire sorting process and only the frequency modulation switches periodically. The on period has a length of $t_{\mathrm{ON}}=1 / \Delta f$, guaranteeing that the pressure nodes move half a wavelength, as illustrated in Fig. 3(c). The off period allows the particles to reach an equilibrium position at the focusing node (small particles) or at the sorting node close to the target outlet (large particles) as shown in Fig. 3(c). This on-off switching approach makes the sorting technique repeatable, as the oscillating small particles are forced to a fixed position periodically. We uploaded to the signal generator one period of the modulation pattern seen in Fig. 3(a) on the right and used internal frequency modulation, with modulation frequency $f_{\mathrm{mod}}=1 /\left(t_{\mathrm{ON}}+t_{\mathrm{OFF}}\right)$. The left column of Fig. 3 corresponds to upwards sorting, while the right column denotes downwards sorting.

COMSOL computational fluid dynamics (CFD) was utilized to simulate the focusing of the particles at different positions within the PDMS microchannel. The details can be found in the supplementary material.

Particles of various sizes and densities/compressibilities were used experimentally. The sorting quality was assessed according to the following figures of merit: ${ }^{16}$

$$
\text { efficiency }=\frac{\text { number of target particles in the target region }}{\text { total number of target particles }},
$$

efficiency

purity $=\frac{\text { efficiency }+ \text { non-target particles in the target region to the total number of non-target particles }}{\text {. }}$
The fabricated microfluidic device presented an inhomogeneous pressure distribution along the SAW active area. The measured ${ }^{11}$ spatial variation in pressure was $30 \%$ higher at the sides of the active area than at the middle, where the average particle speed characterization experiments were carried out. Therefore, the frequency differences in the sorting experiments were increased by $40 \%$ compared to the values suggested in Fig. 2.

The experimental parameters and results are summarized in Table I. For the size-based sorting experiments, the particles were suspended in polyethylene glycol solution (PEG, $0.1 \% \mathrm{w} / \mathrm{v}$ in DI water) to avoid stiction of particles to sidewalls. The particle concentration was at least $2 \cdot 10^{6} \mathrm{ml}^{-1}$, and at least 100 particles were counted to have accurate efficiency and purity values. Five counting periods were randomly chosen and averaged within a $10 \mathrm{~min}$ timeframe when the experiment was running. The voltage used in the experiments was 19-23 $\mathrm{V}_{\mathrm{pk}-\mathrm{pk}}$; lower values did not provide high enough acoustic force to reliably trap and manipulate the particles; higher values result in heat generation that is unfavored for biological applications.

As detailed in the supplementary material, different sorting scenarios are equivalent when the particles to be separated have the same size ratio. For the particle size ratio greater than 1.3, high efficiency and purity, both for the upwards and downwards sorting, were recorded. In all these cases, the efficiency was higher than $84 \%$ and the purity was higher than $81 \%$. The efficiency for both upwards and downwards sorting drops to around $70 \%$ with the purity being approximately $75 \%$ when the particle size ratio decreased to 1.2. As $70 \%-75 \%$ efficiency and purity can be treated as minimum desirable values, the limit of this separation method and device is therefore found to be size ratio of 1.2. Overlay images illustrating the sorting are shown in Fig. 4.

Since the acoustic radiation force depends also on the particle density and compressibility, we carried out separation experiments for $10 \mu \mathrm{m}$ particles of polystyrene, PS $\left(\rho=1.05 \mathrm{~g} / \mathrm{cm}^{3}\right.$ and compressibility $\left.\kappa=250 \mathrm{TPa}^{-1}\right)$, ironoxide, $\mathrm{FeO} \quad\left(\rho=1.5 \mathrm{~g} / \mathrm{cm}^{3}\right.$ and $\left.\kappa<15 \mathrm{TPa}^{-1}\right)$, and poly(methyl methacrylate), PMMA $\left(\rho=1.2 \mathrm{~g} / \mathrm{cm}^{3}\right.$ and $\left.\kappa=170 \mathrm{TPa}^{-1}\right){ }^{17}$ To reduce sedimentation before entering the channel, these particles were suspended in $30 \%(\mathrm{w} / \mathrm{v})$ iodixanol solution (from OptiPrep density gradient, SigmaAldrich, and DI water). The PS and iron-oxide particles showed excellent separability, as shown in Table I, with $>97 \%$ efficiency and $>91 \%$ purity for both sorting

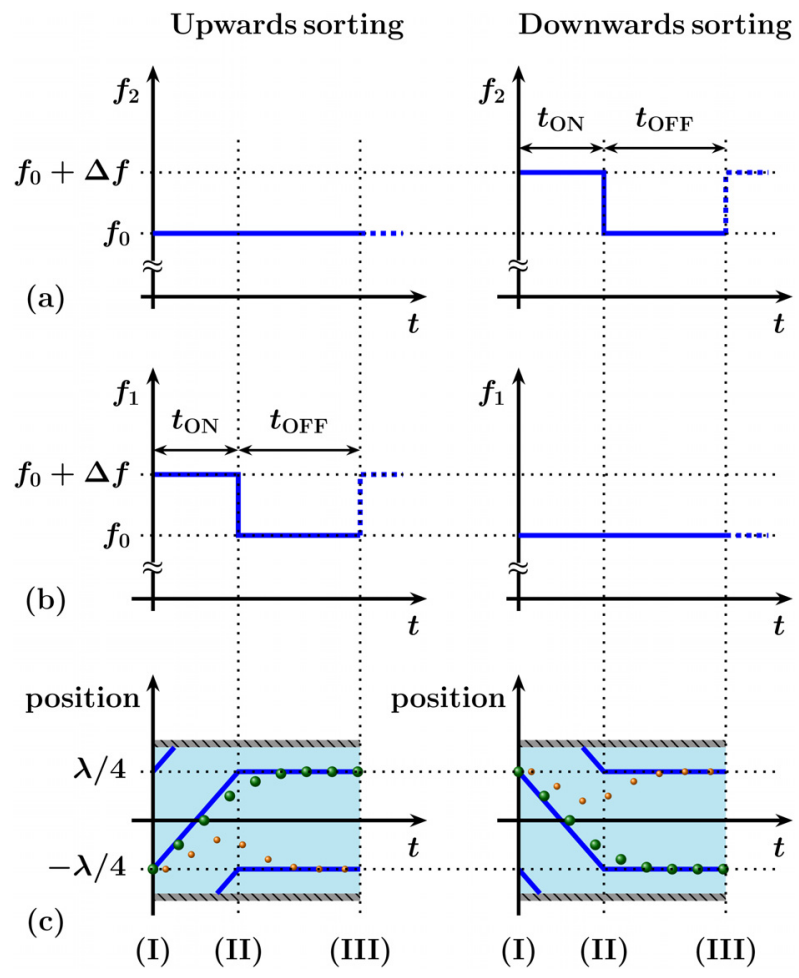

FIG. 3. Detailed illustration of the sorting principle. The left column corresponds to upwards sorting and the right column to downwards sorting. Rows (a) and (b) display the frequency patterns of the two transducers. Row (c) shows the resulting movement of the standing wave (blue) and particles (green and orange balls) within the channel. The particles start at the trapping node, denoted by (I). After the ON period, they are located on different sides of the pressure antinode (II). Therefore, they relax towards different nodes (III). 
TABLE I. Experimental results for various particles and sorting scenarios.

\begin{tabular}{|c|c|c|c|c|c|c|c|c|c|}
\hline \multirow[b]{2}{*}{ Particle mixture } & \multirow[b]{2}{*}{$\operatorname{Dir}^{\mathrm{a}}$} & \multicolumn{3}{|c|}{ Flow rates $(\mu \mathrm{l} / \mathrm{min})$} & \multirow[b]{2}{*}{ Voltage (Vpk-pk) } & \multirow[b]{2}{*}{$\Delta f(\mathrm{~Hz})$} & \multirow[b]{2}{*}{ Off time (s) } & \multirow[b]{2}{*}{ Efficiency (\%) } & \multirow[b]{2}{*}{ Purity (\%) } \\
\hline & & Top inlet & Middle inlet & Bottom inlet & & & & & \\
\hline \multirow[t]{2}{*}{14.5 and $10 \mu \mathrm{m}$ PS } & $\mathrm{D}$ & 0.5 & 0.4 & 1.2 & 19 & -1.3 & 2 & $94 \pm 2$ & $87 \pm 4$ \\
\hline & $\mathrm{U}$ & 1.4 & 0.3 & 0.3 & 19 & 1.4 & 4 & $94 \pm 2$ & $81 \pm 6$ \\
\hline \multirow[t]{2}{*}{8 and $6 \mu \mathrm{m}$ PS } & $\mathrm{D}$ & 0.25 & 0.15 & 0.6 & 19 & -1.2 & 3.5 & $85 \pm 4$ & $83 \pm 5$ \\
\hline & $\mathrm{U}$ & 0.9 & 0.16 & 0.2 & 19 & 1.15 & 4 & $84 \pm 4$ & $81 \pm 7$ \\
\hline \multirow[t]{2}{*}{5 and $6 \mu \mathrm{m}$ PS } & $\mathrm{D}$ & 0.1 & 0.1 & 0.5 & 23 & -1.5 & 3 & $71 \pm 5$ & $78 \pm 6$ \\
\hline & $\mathrm{U}$ & 0.7 & 0.2 & 0.2 & 23 & 1.2 & 3.5 & $67 \pm 9$ & $73 \pm 9$ \\
\hline \multirow[t]{2}{*}{$10 \mu \mathrm{m}$ PS and $\mathrm{FeO}$} & $\mathrm{D}$ & 1.1 & 0.4 & 0.3 & 23 & -2.5 & 1.2 & $97 \pm 4$ & $93 \pm 5$ \\
\hline & $\mathrm{U}$ & 0.3 & 0.4 & 1.1 & 23 & 2.5 & 1.2 & $98 \pm 3$ & $91 \pm 5$ \\
\hline \multirow[t]{4}{*}{$10 \mu \mathrm{m}$ PMMA and $\mathrm{FeO}$} & $\mathrm{D}$ & 1.3 & 0.2 & 0.5 & 19 & -2 & 2 & $85 \pm 7$ & $94 \pm 3$ \\
\hline & & 1.3 & 0.2 & 0.5 & 19 & 1 & 1.5 & $99 \pm 1$ & $71 \pm 3$ \\
\hline & $\mathrm{U}$ & 0.5 & 0.15 & 1.6 & 19 & -2 & 2 & $91 \pm 8$ & $86 \pm 10$ \\
\hline & & 0.5 & 0.15 & 1.6 & 19 & 1 & 1.5 & $97 \pm 3$ & $62 \pm 9$ \\
\hline
\end{tabular}

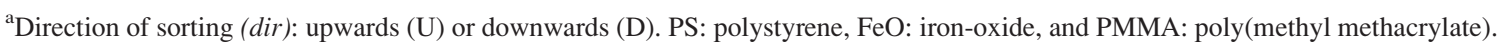

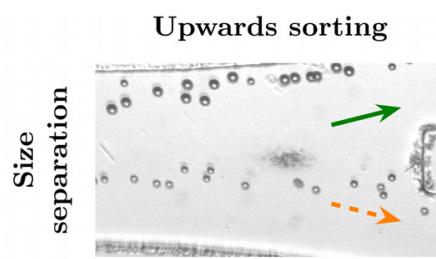

(a)

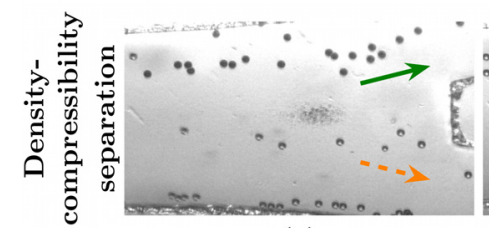

(c)
Downwards sorting

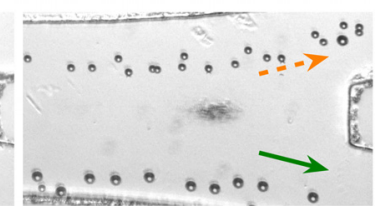

(b)

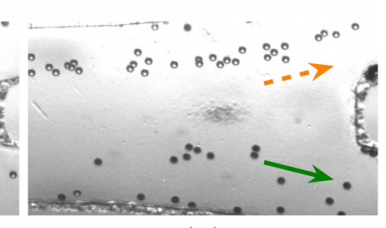

(d)
FIG. 4. (a) and (b) Overlay images of size-based sorting for 10 and $14.5 \mu \mathrm{m}$ PS particles in diameter. (c) and (d) Overlay images of density/compressibility particle sorting of PS (appearing with white center) and iron-oxide particles (appearing as solid black), $10 \mu \mathrm{m}$ in diameter. For sorting parameters, refer to Table I. Green solid and orange dashed arrows indicate target and waste particle flow, respectively.

directions. With the reduced difference in density for the PMMA and iron-oxide particles, high efficiency and purity were achieved by using two different frequency modulations: for $1 \mathrm{~Hz}$, high efficiency ( $>97 \%$ ), and for $2 \mathrm{~Hz}$, high purity $(>86 \%)$ were measured. For this sorting scenario, both figures of merit are lower, and their variation is higher for the upwards sorting. Our previous works ${ }^{10,11}$ also investigated sorting based on density and compressibility differences of particles. Although they showed higher efficiency values, they were carried out in the absence of flow. Therefore, no issues were present such as hydrodynamic focusing inaccuracies or the particles being subjected to the acoustic field for slightly different periods of time due to the parabolic flow profile and travel time though the device.

Similar acoustic methods achieving particle or cell separation of similar size or physical properties are listed in Table II with the respective figures of merit. Most of the works only present a single efficiency value for characterizing the device, and therefore, direct comparison with our method is difficult, since achieving high efficiency is possible even with extremely low purity. Nevertheless, in all cases, our method shows superiority in both the figures of merit and particle size ratio.

Sensitivity analysis of the device was carried out by varying the flow rates at the various inlets for 10 and $14.5 \mu \mathrm{m}$ diameter particles. As a reference, we used flow rates corresponding to the best performance sorting scenario and varied the sheath inflows, from $0.2 \mu \mathrm{l} / \mathrm{min}$ less than the reference to $0.2 \mu \mathrm{l} / \mathrm{min}$ above the reference. When examining only the trapping performance of the device, at least $97 \%$ of the particles were trapped towards the non-sorting outlet. The 3\% particle loss was observed for low sheath or high particle flow and was due to acoustic streaming at the channel walls, trapping particles towards the walls. ${ }^{17}$ Sorting

TABLE II. Comparison of the results with other acoustic sorting methods. E denotes the efficiency and P denotes the purity.

\begin{tabular}{|c|c|c|c|}
\hline Reference & Device parameters & Particles or cells to be separated & Figure of merit \\
\hline ON-OFF frequency switch (this work) & $\begin{array}{l}\text { 13.3 MHz, } 50 \mu \mathrm{m} \text { channel height, } \\
240 \mu \mathrm{m} \text { channel width }\end{array}$ & $\begin{array}{c}14.5 \mu \mathrm{m} / 10 \mu \mathrm{m} \text { PS, size ratio } 1.45 \\
10 \mu \mathrm{m} \text { PS and iron-oxide } \\
10 \mu \mathrm{m} \text { PMMA and } \mathrm{FeO}\end{array}$ & $\begin{array}{c}\mathrm{E}: 94 \pm 2 \%, \mathrm{P}: 87 \pm 4 \%, 81 \pm 6 \% \\
\mathrm{E}:<98 \pm 3 \%, \mathrm{P}: 93 \pm 5 \% \\
\mathrm{E}:<99 \pm 1 \%, \mathrm{P}: 94 \pm 3 \%\end{array}$ \\
\hline $\begin{array}{l}\text { Continuously phase modulated by the } \\
\text { frequency step }\end{array}$ & $\begin{array}{c}14 \mathrm{MHz}, 1050 \mu \mathrm{m} \text { channel width, } 80 \\
\mu \mathrm{m} \text { height }\end{array}$ & $\begin{array}{c}15 \mu \mathrm{m} / 6 \mu \mathrm{m} \text { PS, size ratio } 2.5 \\
2 \mu \mathrm{m} \text { PS particles, HaCaT cells } \\
(\text { mean dia } 24.47 \mu \mathrm{m} \text { ), size ratio }>10\end{array}$ & $\begin{array}{l}\text { E: } 72 \%, P: n / a \\
\text { E: } 83 \%, P: n / a\end{array}$ \\
\hline Standing wave sorter ${ }^{18}$ & $\begin{array}{c}13.2 \mathrm{MHz}, 300 \mu \mathrm{m} \text { width, } 100 \mu \mathrm{m} \\
\text { height }\end{array}$ & $\begin{array}{c}3 \text { and } 10 \mu \mathrm{m} \text { PS, size ratio } 3.33 ; 3 \\
\text { and } 5 \text { PS, size ratio } 1.67\end{array}$ & $\begin{array}{c}\text { E: for } 3 \mu \mathrm{m} 87.4 \%-94.8 \% \text {; for } 10 \\
\mu \mathrm{m} 94.6 \%-100 \%, \text { no data for } 3 / 5 \mu \mathrm{m}\end{array}$ \\
\hline Standing wave sorter ${ }^{19}$ & $\begin{array}{c}13.3 \mathrm{MHz}, \begin{array}{c}150 \mu \mathrm{m} \text { width, } 80 \mu \mathrm{m} \\
\text { height }\end{array}\end{array}$ & $\begin{array}{l}10 \mu \mathrm{m} \text { PS }\left(1.05 \mathrm{~g} / \mathrm{cm}^{3}\right) \text { and mela- } \\
\text { mine }\left(1.71 \mathrm{~g} / \mathrm{cm}^{3}\right)\end{array}$ & E: $87.2 \%-98.8 \%, P: \mathrm{n} / \mathrm{a}$ \\
\hline
\end{tabular}


TABLE III. Experimental results for flow sensitivity analysis for 10 and $14.5 \mu \mathrm{m}$ diameter particles. The experiments were conducted with the same excitation signals reported in Table I.

\begin{tabular}{lccccc}
\hline \hline & \multicolumn{3}{c}{ Flow rates $(\mu \mathrm{l} / \mathrm{min})$} & & \\
\cline { 2 - 4 } Dir $^{\mathrm{a}}$ & Top inlet & Middle inlet & Bottom inlet & Efficiency (\%) & Purity (\%) \\
\hline $\mathrm{D}^{\mathrm{b}}$ & 0.5 & 0.4 & 1.2 & 94 & 87 \\
$\mathrm{D}$ & 0.3 & 0.4 & 1.0 & 85 & 87 \\
$\mathrm{D}$ & 0.7 & 0.4 & 1.4 & 88 & 83 \\
$\mathrm{U}^{\mathrm{b}}$ & 1.4 & 0.3 & 0.3 & 94 & 81 \\
$\mathrm{U}$ & 1.2 & 0.3 & 0.1 & 84 & 84 \\
$\mathrm{U}$ & 1.6 & 0.3 & 0.5 & 73 & 84 \\
\hline \hline
\end{tabular}

${ }^{\text {a }}$ Direction of sorting (Dir): upwards (U) or downwards (D).

${ }^{\mathrm{b}}$ Reference experiments, as shown in Table I.

experiments for various flow rates are listed in Table III. When the sheath flow on the non-target outlet side was small, particles attached to the sidewall due to acoustic streaming. Interestingly, even for increased sheath flows, the efficiency dropped. We attribute this decrease in efficiency to the faster transport of particles and therefore not enough time spent in the channel for sorting. Upwards sorting was more susceptible to variations, which suggests that the asymmetric inlet design favors the more natural focusing at the top node and sorting towards the bottom outlet.

In conclusion, we presented an easily reconfigurable method for particle separation in surface wave microfluidic devices increasing the performance of acoustic sorting techniques that are based on the frequency difference between two transducers. ${ }^{9}$ Bidirectional sorting has been demonstrated by adjusting the inflow rates and electrical signal of transducers. The separation distance achieved with this method is half the wavelength, which is double that of conventional time-of-flight methods. This technique can be applied for various particle separation scenarios due to its versatility, reconfigurability, and simple electrical excitation requirements. This technique can also be scaled easily, and adjusting the frequency is straightforward given the scaling laws in the supplementary material. Future work will analyze the application of the method for biological living cell separation.

See supplementary material for details on the derivation of acoustic radiation force in quasi-standing waves, simulation of the velocity profile and particle focusing within the microchannel, and scaling of the optimal sorting frequency range for various experimental parameters.

The authors would like to thank Mr. Neil Ross for his help with the cleanroom device fabrication processes. We also would like to thank Dr. William N. MacPherson for access to thermal evaporator equipment. We are grateful to Dr. Muhsincan Sesen and Mr. Gergely Hantos for their suggestions with the microfluidic setup.

A.B. acknowledges support from Heriot-Watt University. J.R. acknowledges the University of Glasgow Lord Kelvin and Adam Smith Research Fellowship.

${ }^{1}$ C. W. Shields, C. D. Reyes, and G. P. Lopez, Lab Chip 15, 1230 (2015).

${ }^{2}$ T. Laurell, F. Petersson, and A. Nilsson, Chem. Soc. Rev. 36, 492 (2007).

${ }^{3}$ J. Shi, H. Huang, Z. Stratton, Y. Huang, and T. J. Huang, Lab Chip 9, 3354 (2009).

${ }^{4}$ X. Y. Ding, Z. L. Peng, S. C. S. Lin, M. Geri, S. X. Li, P. Li, Y. C. Chen, M. Dao, S. Suresh, and T. J. Huang, Proc. Natl. Acad. Sci. U. S. A. 111, 12992 (2014).

${ }^{5}$ G. Whitworth, M. A. Grundy, and W. T. Coakley, Ultrasonics 29, 439 (1991).

${ }^{6}$ T. Kozuka, T. Tuziuti, H. Mitome, F. Arai, and T. Fukuda, in Mhs 2000: Proceedings of the 2000 International Symposium on Micromechatronics and Human Science (2000), p. 201.

${ }^{7}$ W. T. Coakley, G. Whitworth, M. A. Grundy, R. K. Gould, and R. Allman, Bioseparation 4, 73 (1994).

${ }^{8} \mathrm{~S}$. Peterson, G. Perkins, and C. Baker, in Annual Conference of the Engineering in Medicine and Biology Society (1986), pp. 154.

${ }^{9}$ J. Lee, C. Rhyou, B. Kang, and H. Lee, J. Phys. D: Appl. Phys. 50, 165401 (2017).

${ }^{10}$ M. A. B. Andrade, G. D. Skotis, S. Ritchie, D. R. S. Cuming, M. O. Riehle, and A. L. Bernassau, IEEE Trans. Ultrason. Ferroelectr. Freq. Control 63, 1593 (2016).

${ }^{11}$ G. Simon, M. A. B. Andrade, J. Reboud, J. Marques-Hueso, M. P. Y. Desmulliez, J. M. Cooper, M. O. Riehle, and A. L. Bernassau, Biomicrofluidics 11, 054115 (2017).

${ }^{12}$ N. D. Orloff, J. R. Dennis, M. Cecchini, E. Schonbrun, E. Rocas, Y. Wang, D. Novotny, R. W. Simmonds, J. Moreland, I. Takeuchi, and J. C. Booth, Biomicrofluidics 5, 44107 (2011).

${ }^{13}$ F. Petersson, A. Nilsson, C. Holm, H. Jönsson, and T. Laurell, Lab Chip 5, 20 (2005).

${ }^{14}$ H. Bruus, Lab Chip 12, 1578 (2012).

${ }^{15}$ J. Leach, H. Mushfique, S. Keen, R. Di Leonardo, G. Ruocco, J. M. Cooper, and M. J. Padgett, Phys. Rev. E: Stat., Nonlinear, Soft Matter Phys. 79, 026301 (2009).

${ }^{16}$ A. Bussonniere, Y. Miron, M. Baudoin, O. Bou Matar, M. Grandbois, P. Charette, and A. Renaudin, Lab Chip 14, 3556 (2014).

${ }^{17}$ N. Nama, R. Barnkob, Z. Mao, C. J. Kahler, F. Costanzo, and T. J. Huang, Lab Chip 15, 2700 (2015).

${ }^{18}$ R. Guldiken, M. C. Jo, N. D. Gallant, U. Demirci, and J. Zhe, Sensors (Basel) 12, 905 (2012).

${ }^{19}$ M. C. Jo and R. Guldiken, Sens. Actuators, A 187, 22 (2012). 